\title{
DOUBLE COSET MATRICES AND GROUP CHARACTERS
}

\section{J. S. FRAME}

1. Introduction. The principal theorem of this paper extends to the characters of the irreducible representations of an intransitive group a theorem proved in an earlier paper by the author ${ }^{1}$ for the degrees of the irreducible representations of a transitive group. A by-product of the development is the theorem that the sum of the traces of the permutations of any subgroup of a permutation group is not less than the corresponding sum for one of its cosets.

Every finite permutation group $G$, of order $g$ and degree $n$, can be written as a group of permutation matrices. The $n \times n$ matrix $R(\gamma)$ which corresponds to the element $\gamma$ of $G$ can be written as a direct sum of submatrices $R^{t}(\gamma)$ of $n^{t}$ dimensions corresponding to the $n^{t}$ symbols of a transitive constituent $C^{t}$ of $G .{ }^{2}$ Associated with such a transitive constituent $C^{t}$ is a class of conjugate subgroups, $H_{r}^{t}=\left(\gamma_{\tau}^{t}\right)^{-1} H^{t} \gamma_{r}^{t}$, each of order $h^{t}$, of which $H^{t}$ shall be the subgroup leaving fixed the first symbol of $C^{t}$, and $H_{\tau}^{t}$ the subgroup leaving fixed the $\tau$ th symbol. If $\gamma_{\alpha}$ is any element of $G$, then in the set of $h^{8} h^{t}$ group elements $H^{s} \gamma_{\alpha} H^{t}$, each element will appear $h_{\alpha}^{s t}$ times, where $h_{\alpha}^{s t}$ is the order of the cross-cut of the subgroups $H_{\alpha}^{s}=\gamma_{\alpha}^{-1} H^{s} \gamma_{\alpha}$ and $H^{t}$.

Counting each element of the set just once, we define the double coset $H_{\alpha}^{s t}$ by the formula

$$
H_{\alpha}^{s t}=H_{\gamma_{\alpha}}^{s} H^{t} / h_{\alpha}^{s t} .
$$

Any element from a double coset can be chosen as the defining element $\gamma_{\alpha}$. The inverses of the elements of a double coset $H_{\alpha}^{s t}$ themselves form a double coset which we call the inverse double coset and denote by $H_{\alpha^{\prime}}^{t s}$. The product of two double cosets is a linear combination of double cosets. By considering $H_{\alpha}^{r s}$ as a sum of $h^{r} / h_{\alpha}^{r s}$ left cosets of $H^{s}$, and $H_{\beta^{\prime}}^{s t}$ as a sum of $h^{t} / h_{\beta^{\prime}}^{s t}$ right cosets of $H^{s}$, and noting that $H^{s} H^{s}=h^{s} H^{s}$, it is apparent that each element in the product $H_{\alpha}^{r s} H_{\beta^{\prime}}^{s t}$ occurs a multiple of $h^{s}$ times. We define the positive integers $c_{\alpha \beta \eta}^{r s t}$ by the formula

$$
H_{\alpha}^{r s} H_{\beta^{\prime}}^{s t} / h^{s}=\sum_{\eta} c_{\alpha \beta \eta}^{r s t} H_{\eta}^{r t} .
$$

Presented to the Society, April 3, 1942; received by the editors April 4, 1942.

1 J. S. Frame, The double cosets of a finite group, Bull. Amer. Math. Soc. vol. 47 (1941) p. 459.

${ }^{2}$ Throughout this paper the superscripts will refer to the transitive constituents. 
Let $\mu^{s t}$ be the number of double cosets $H_{\alpha}^{s t}$ for fixed $s, t$. Then for fixed $\eta$ the constants $c_{\alpha \beta \eta}^{r s t}$ are elements of a $\mu^{r s}$ - by $\mu^{s t}$-dimensional rectangular matrix $M^{s}\left(H_{\eta}^{n t}\right)$, and the transposed matrix is the matrix $M^{s}\left(H_{\eta^{\prime}}^{t r}\right)$ for the inverse double coset. For $r=t$ these matrices are square. Their sum, with parameters $b_{\eta}^{t}$ as coefficients, defines the important matrix

$$
K^{s t}=\sum_{\eta} b_{\eta}^{t} M^{s}\left(H_{\eta}^{t t}\right)
$$

each of whose elements are linear combinations of the parameters $b_{\eta}^{t}$.

The principal theorem of this paper states that the determinant of the matrix $n^{t} K^{s t}$ splits into factors $\chi_{i}$ which are linear in the parameters $b_{\eta}$, that these factors $\chi_{i}$ are essentially the characters of the irreducible representations $\Gamma_{i}$ of $G$, and that the multiplicity $m_{i}$ of the linear factor $\chi_{i}$ is $\mu_{i}^{s} \mu_{i}^{t}$, where $\mu_{i}^{t}$ is the multiplicity with which $\Gamma_{i}$ occurs in the reduction of the transitive group of matrices $R^{t}(\gamma)$, and where $\sum \mu_{i}^{s} \mu_{i}^{t}=\mu^{s t}$. Finally the numerical coefficient which appears is an integer divisible by $n^{s} n^{t}$. Written as a formula, the theorem reads

(1.4) ThEOREM.

$$
\left(n^{t}\right)^{m}\left|K^{s t}\right|=n^{s} n^{t} A^{s t} \prod_{i}\left(\chi_{i}\right)^{m_{i}}
$$

where $m_{i}=\mu_{i}^{s} \mu_{i}^{t}, m=\sum m_{i}=\mu^{s t}, A^{s t}=$ an integer.

A more precise definition of $\chi_{i}$ is obtained after writing

$$
b_{\eta}^{t}=\sum_{\lambda} k_{\eta \lambda}^{t} a_{\lambda}
$$

where $a_{\lambda}$ are new arbitrary parameters and $k_{\eta \lambda}^{t}$ is the number of elements of the double coset $H_{\eta}^{t t}$ which belong to the class of conjugates $C_{\lambda}$. Then $\chi_{i}=\chi_{i}\left(\sum a_{\lambda} C_{\lambda}\right)$ is defined as the character of the ring element $\sum a_{\lambda} C_{\lambda}$ in the representation $\Gamma_{i}$. The integer $A^{s t}$ enters in the proof as the squared absolute value of the determinant of a certain transformation matrix. It is not necessarily a rational square.

In the special case of this theorem which appeared in the earlier paper already cited, ${ }^{3}$ all parameters $a_{\lambda}$ except $a_{1}$ were zero, $s$ was equal to $t, \chi_{i}$ was the degree $n_{i}$, and the matrix $K^{s t}$ was a diagonal matrix with elements there denoted by $k_{t}$.

In $\$ 2$ the matrices of an intransitive group are broken up into blocks corresponding to the transitive constituents, and it is proved that the sum of the traces of the matrices of a subgroup is not less

${ }^{3}$ J. S. Frame, loc. cit. 
than the corresponding sum for a coset. In $\$ 3$ a set of "double coset matrices" $V_{\alpha}^{t s}$, closely related to the double cosets $H_{\alpha}^{s t}$, are found to form a basis for the matrices $V$ permutable with a group of permutation matrices. Their products are expressible in terms of the structure constants $c_{\alpha \beta \eta}^{r s t}$ already defined in (1.2). The matrix $R(a)$ corresponding to the ring element $a=\sum a_{\lambda} C_{\lambda}$ is expressed in terms of the double coset matrices, and used in $\$ 4$ to build a matrix of traces $\chi\left(V_{\alpha^{\prime}}^{t s} V_{\beta}^{s t} R(a)\right)$ which is shown to be precisely the matrix $n^{t} K^{s t}$ defined by (1.3). A unitary change of coordinates in $\$ 5$ induces a change of basis from the matrices $V_{\alpha}^{t s}$ to new basis matrices $E_{\eta}^{t s}$ by means of a transformation $\left(\rho_{\alpha \eta}^{s t}\right)$ whose determinant enters in the final factoring of the determinant $n^{t} K^{s t}$ from which the group characters are obtained. An example to show the application of the main theorem is given in $\$ 6$.

2. Cosets and submatrices. The $n$ symbols of the intransitive group $G$ will be assumed to be collected together into transitive constituents $C^{t}$ of degree $n^{t}$, and the $n$ right cosets $H^{t} \gamma_{\tau}^{t}$ will be arranged, in an order which preserves this grouping, so as to form the elements of a basis vector $b$ of $n$ rows and one column. Multiplication of $b$ on the right by an element $\gamma$ of $G$ permutes these cosets among themselves within each transitive constituent, so a permutation matrix $R(\gamma)$ is defined by the equation

$$
b \gamma=R(\gamma) b .
$$

Similarly if $b^{\prime}$ and $R^{\prime}(\gamma)$ are the transposes of $b$ and $R(\gamma)$, and if $E$ is the $n \times n$ unit matrix, we have

$$
b^{\prime}(\gamma E)=b^{\prime} R^{\prime}(\gamma)=b^{\prime} R\left(\gamma^{-1}\right) .
$$

Let us define the idempotent matrix $E^{t}$ to be the $n \times n$ matrix which is the unit matrix for the symbols of the constituent $C^{t}$ and the zero matrix for the other symbols, and let us define a "completely symmetric" $n \times n$ matrix $W$ each of whose $n^{2}$ elements is a 1 . Then

$$
E=\sum_{t} E^{t}, \quad E^{t} E^{t}=E^{t}, \quad E^{t} E^{s}=0 \text { if } t \neq s .
$$

Let us further define the block $C^{t s}$ to be the $n^{t} \times n^{s}$ rectangular submatrix of an $n \times n$ matrix, whose rows correspond to the symbols of $C^{t}$ and whose columns correspond to the symbols of $C^{s}$. We see that the matrix

$$
W^{t s}=E^{t} W E^{s}
$$

consists of 1's in the block $C^{t s}$ and 0's elsewhere.

The general matrix $R(\gamma)$ of the intransitive permutation group $G$ 
may be broken up into transitive components $R^{t}(\gamma)$ in the diagonal blocks $C^{t t}$. We define

$$
R^{t}(\gamma)=E^{t} R(\gamma)=R(\gamma) E^{t}
$$

and we then have

$$
R(\gamma)=\sum_{t} R^{+}(\gamma)
$$

The fact that the group $G$ is transitive in the symbols of the constituent $C^{t}$ shows that the sum of the matrices $R^{t}(\gamma)$ for all $\gamma$ in $G$, which we denote by $R^{t}(G)$, is a multiple of the matrix $W^{t}$. A simple count proves the following relation:

LEMMA.

$$
R^{t}(G)=h^{t} W^{t t} .
$$

A theorem on the traces of subgroups and cosets, suggested to the author by G. Polya as having been experimentally verified but not theoretically proved, is a consequence of this lemma.

(2.8) THEOREM. The sum of the numbers of symbols left fixed by the individual permutations of a subgroup of any permutation group is greater than or equal to the corresponding sum for the permutations of any coset of that subgroup.

To prove this theorem we note first that each specified sum is the sum of traces of the elements of a subgroup $H$ or of a coset $H \gamma$, and second that if the theorem holds for every transitive constituent of an intransitive group $G$, then it holds, by addition, for $G$ as well. Focusing attention on a constituent $C^{t}$ which is transitive for $G$, we further subdivide it into sets $C^{t u}$ which are transitive for the given subgroup $H$. To the corresponding matrices $R^{t u}(H)$ we apply the lemma, showing that each is a square block of dimension $n^{t u}$, each of whose elements is equal to $h / n^{t u}$, and whose trace is equal to $h$. Multiplication of the matrix $R^{t}(H)$ by a matrix $R(\gamma)$ has the effect of permuting the columns and replacing the diagonal elements either by the same positive integer as before, or else by 0 . Thus the trace of $R^{t}(H \gamma)$ is less than or equal to the trace of $R^{t}(H)$, which was to be proved.

3. Double coset matrices and structure constants. To describe the structure of the general $n$-dimensional matrix $V$ which is permutable with every permutation matrix $R(\gamma)$, of an intransitive permutation group $G$, we define 


$$
V^{t s}=E^{t} V E^{s} .
$$

Then the permutability condition

$$
V R(\gamma)=R(\gamma) V
$$

together with (2.5) implies the relations

$$
V^{t s} R^{s}(\gamma)=E^{t} V R(\gamma) E^{s}=E^{t} R(\gamma) V E^{s}=R^{t}(\gamma) V^{t s},
$$

which may be expressed in words by saying that the rectangular submatrix $V^{t s}$ of $V$ intertwines the matrices $R^{s}(\gamma)$ and $R^{t}(\gamma)$. From (3.3) we obtain the equation

$$
V^{t s}=R^{t}(\gamma) V^{t s} R^{s}\left(\gamma^{-1}\right),
$$

which states that the rectangular matrix $V^{t s}$ is unchanged if its rows are permuted by the matrix $R^{t}(\gamma)$ while its columns undergo simultaneously the transposed permutation $\left[R^{s}(\gamma)\right]^{\prime}$. It follows in general that many of the coefficients in the matrix $V^{t s}$ are necessarily equal "by symmetry." If $E_{\tau \sigma}^{t s}$ is defined to be a matrix which is zero except for a 1 in the $\tau$ th row and the $\sigma$ th column of the block $C^{t s}$, if $H^{t}, \gamma_{r}^{t}$, $H^{s}, \gamma_{\sigma}^{s}$ are defined as in $\S 1$, and $\theta^{t}$ and $\theta^{s}$ are elements of $H^{t}$ and $H^{s}$, respectively, then

$$
E_{\tau \sigma}^{t_{s}}=R^{t}\left(\theta^{t} \gamma_{\tau}^{t}\right)^{-1} E_{11}^{t_{s}} R^{s}\left(\theta^{s} \gamma_{\sigma}^{s}\right)
$$

To obtain an invariant matrix we take the sum of all the transforms of (3.5) by all $\gamma$ in $G$. We get

$$
\begin{aligned}
\sum_{\gamma \in G} R^{t}\left(\gamma^{-1}\right) E_{\tau \sigma}^{t s} R^{s}(\gamma) & =\sum_{\gamma} R^{t}\left(\theta^{t} \gamma_{\tau}^{t} \gamma\right)^{-1} E_{11}^{t s} R^{s}\left(\theta^{s} \gamma_{\sigma}^{s} \gamma\right) \\
& =\sum_{\delta} R^{t}\left(\delta^{-1}\right) E_{11}^{t s} R^{s}\left(\theta^{s} \gamma_{\sigma}^{s}\left(\gamma_{\tau}^{t}\right)^{-1}\left(\theta^{t}\right)^{-1}\right) R^{s}(\delta) \\
& =\sum_{\gamma} R^{t}\left(\gamma^{-1}\right) E_{11}^{t s} R^{s}\left(\gamma_{\alpha}\right) R^{s}(\gamma)
\end{aligned}
$$

where $\gamma_{\alpha}$ is any element chosen from the double coset $H_{\alpha}^{s t}$ which contains $\gamma_{\sigma}^{s}\left(\gamma_{\tau}^{t}\right)^{-1}$. We define the double coset matrix $V_{\alpha}^{t s}$ by the formula

$$
V_{\alpha}^{t s}=\left(1 / h_{\alpha}^{s t}\right) \sum_{\gamma \in G} R^{t}\left(\gamma^{-1}\right) E_{11}^{t s} R^{s}\left(\gamma_{\alpha}\right) R^{s}(\gamma),
$$

so that its coefficients are all 0 's and 1's. In this matrix there are $h^{t} / h_{\alpha}^{s t} 1$ 's in each row and $h^{s} / h_{\alpha}^{s t} 1$ 's in each column of the block $C^{t s}$, and 0 's elsewhere. Furthermore, by averaging equation (3.4) for all $\gamma$ in $G$, it is readily seen that every $V^{t s}$ is a linear combination of these basis matrices $V_{\alpha}^{t s}$, and in particular that 


$$
\sum_{\alpha} V_{\alpha}^{t s}=W^{t_{s}}
$$

From this discussion it also follows that

$$
W^{t s} V_{\alpha}^{s r}=W^{t r} h^{r} / h_{\alpha}^{s r} .
$$

The number of independent matrices $V_{\alpha}^{t s}$ in the block $C^{t s}$ is equal to the number of double cosets $H_{\alpha}^{s t}$, which was denoted by $\mu^{s t}$. Since the transposed matrix $\left(V_{\alpha}^{t s}\right)^{\prime}=V_{\alpha^{\prime}}^{s t}$ corresponds to the inverse double coset $\left(H_{\alpha}^{s t}\right)^{-1}=H_{\alpha^{\prime}}^{t s}=H^{t} \gamma_{\alpha}^{-1} H^{s} / h_{\alpha}^{s t}$, it follows that $\mu^{s t}=\mu^{t s}$.

The product of two double coset matrices $V_{\beta^{\prime}}^{t s}$ and $V_{\alpha}^{s r}$ is given in terms of the coefficients $c_{\alpha \beta \eta}^{r s t}$ of (1.2) as follows:

$$
V_{\beta}^{t^{\prime}} V_{\alpha}^{8 r}=\sum_{\eta} c_{\alpha \beta \eta}^{r s t} V_{\eta}^{t r} .
$$

To prove this we work from the definition (3.7) and apply (1.2)

$$
\begin{aligned}
V_{\beta^{\prime}}^{t s} V_{\alpha}^{s r} & =\left(1 / h_{\alpha}^{r s} h_{\beta}^{s t}\right) \sum_{\gamma, \delta} R^{t}\left(\gamma^{-1}\right) E_{11}^{t s} R^{s}\left(\gamma_{\beta^{\prime}}\right) R^{s}(\gamma) R^{s}\left(\delta^{-1}\right) E_{11}^{s r} R^{r}\left(\gamma_{\alpha}\right) R^{r}(\delta) \\
& =\left(1 / h_{\alpha}^{r s} h_{\beta}^{s t}\right) \sum_{\gamma} R^{t}\left(\gamma^{-1}\right) E_{11}^{t s} E_{11}^{s r} R^{r}\left(\gamma_{\alpha} H^{s} \gamma_{\beta^{\prime}} \gamma\right) \\
& =\left(1 / h^{r} h^{s} h^{t}\right) \sum_{\gamma} R^{t}\left(\gamma^{-1}\right) E_{11}^{t r} R^{r}\left(H_{\alpha}^{r s} H_{\beta^{\prime}}^{s t}\right) R^{r}(\gamma) \\
& =\sum_{\eta} c_{\alpha \beta \eta}^{r s t} \sum_{\gamma} R^{t}\left(\gamma^{-1}\right) E_{11}^{t s} R^{r}\left(H_{\eta}^{r t} / h^{r} h^{t}\right) R^{r}(\gamma) \\
& =\sum_{\eta}^{r s} c_{\alpha \beta \eta}^{r t} V_{\eta}^{t r} .
\end{aligned}
$$

By summing equation (3.10) first for $\beta$ and then for $\alpha$, and applying (3.9) we obtain

Hence

$$
\begin{aligned}
W^{t s} V_{\alpha}^{8 r} & =\sum_{\beta \eta} c_{\alpha \beta \eta}^{r s t} V_{\eta}^{t r}=W^{t r}\left(h^{r} / h_{\alpha}^{s r}\right), \\
V_{\beta}^{t s} W^{s r} & =\sum_{\alpha \eta} c_{\alpha \beta \eta}^{r s t} V_{\eta}^{t r}=\left(h^{t} / h_{\beta}^{t s}\right) W^{t r} .
\end{aligned}
$$

$$
\sum_{\beta} c_{\alpha \beta \eta}^{r s t}=h^{r} / h_{\alpha}^{s r} ; \quad \sum_{\alpha} c_{\alpha \beta \eta}^{r s t}=h^{t} / h_{\beta}^{s t} ; \quad \sum_{\alpha, \beta} c_{\alpha \beta \eta}^{r s t}=n^{s} .
$$

These sums are the sums for a column, or row, or all elements of the matrix $M^{s}\left(H_{\eta}^{n}\right)$ defined in $\S 1$.

4. Conjugate classes and the trace matrix. The matrix $R\left(C_{\lambda}\right)$, defined to be the sum of the matrices $R(\gamma)$ for all $\gamma$ in the class of conjugate elements $C_{\lambda}$, and likewise the more general matrix $R(a)$, 
where $a=\sum a_{\lambda} C_{\lambda}$ is a general element from the central of the group ring of $G$ and $a_{\lambda}$ are arbitrary parameters, are both matrices permutable with the group, and as such are expressible in terms of the double coset matrices. They are confined to the diagonal blocks $C^{t t}$, so we have

$$
R^{t}\left(C_{\lambda}\right)=\sum_{\delta} g_{\delta \lambda}^{t} V_{\delta}^{t t} ; \quad R^{t}(a)=\sum a_{\lambda} R^{t}\left(C_{\lambda}\right) .
$$

The coefficient $g_{\eta \lambda}^{t}$ is the number of elements of $C_{\lambda}$ which belong to any particular right coset of $H_{\eta}^{u}$.

We are now ready to define and evaluate the important $\mu^{8 t}$-dimensional trace matrix $X_{\alpha \beta}^{s t}(a)$ :

$$
\begin{aligned}
& X_{\alpha \beta}^{s t}(a) \equiv \chi\left(V_{\alpha^{\prime}}^{t s} V_{\beta}^{s t} R^{t}(a)\right) \\
& =\chi\left(\sum_{\eta} c_{\alpha \beta \eta}^{t s t} V_{\eta^{\prime}}^{t t} \sum_{\lambda} a_{\lambda} R^{t}\left(C_{\lambda}\right)\right) \\
& =\sum_{\lambda} a_{\lambda} \chi\left(\sum_{\eta, \delta} c_{\alpha \beta \eta}^{t s t} g_{\delta \lambda}^{t} V_{\eta^{\prime}}^{t t} V_{\delta}^{t t}\right) \\
& =\sum_{\lambda, \eta} a_{\lambda} c_{\alpha \beta \eta}^{t s t} g_{\eta \lambda}^{t} \chi\left(V_{\eta^{\prime}}^{t t} V_{\eta}^{t t}\right) \\
& =\sum_{\lambda, \eta} a_{\lambda} c_{\alpha \beta \eta}^{t s t} k_{\eta \lambda}^{t} n^{t} \\
& =n^{t} \sum_{\eta} c_{\alpha \beta \eta}^{t s t} b_{\eta}^{t} .
\end{aligned}
$$

The letter $\chi$ is used to denote the trace of a matrix. The new coefficients $k_{\eta \lambda}^{t}$ and parameters $b_{\eta}^{t}$ are defined as follows:

$$
k_{\eta \lambda}^{t}=g_{\eta \lambda}^{t} h^{t} / h_{\eta}^{t t} ; \quad b_{\eta}^{t}=\sum_{\lambda} a_{\lambda} k_{\eta \lambda}^{t} .
$$

The integer $k_{\eta \lambda}^{t}$ is the number of elements of $C_{\lambda}$ which occur in the whole double coset $H_{\eta}^{t}$. In terms of the matrix $K^{s t}$ of (1.3), we have

$$
X^{s t}(a)=n^{t} K^{s t}(a)=n^{t} \sum_{\eta} b_{\eta}^{t} M^{s}\left(H_{\eta}^{t t}\right) .
$$

In applying the theory to a given group, as in $\S 6$, we first compute the matrices $M^{s}$ for each double coset, form the sum with parameters $b_{\eta}^{t}$ as coefficients, and factor the resulting determinant into factors linear in the $b_{\eta}^{t}$. One of these factors will be $\left(b_{1}^{t}+b_{2}^{t}+\cdots\right)$, corresponding to the identity representation. We then compute the integers $k_{\eta \lambda}^{t}$ by studying the group directly, and replace the parameters 
$b_{\eta}^{t}$ by appropriate linear combinations of the parameters $a_{\lambda}$. The coefficients of $a_{\lambda}$ so obtained in any linear factor, divided by the number $g_{\lambda}$ of elements in $C_{\lambda}$, are proportional to the traces $\chi_{i \lambda}$ of the elements of $C_{\lambda}$ in the irreducible representation $\Gamma_{i}$ corresponding to that factor.

5. The determinant of the trace matrix. A unitary matrix $U$ exists which will simultaneously reduce all the matrices $R(\gamma)$, where $\gamma$ can now be thought of as an arbitrary element of the group ring of $G$. The matrix $U$ can be chosen to be zero outside the diagonal blocks $C^{t t}$, in which we define $U^{t}=E^{t} U E^{t}$. It can further be so chosen that all the equivalent irreducible components in the decomposition of $R(\gamma)$ are actually identical, and we shall assume that this is done, and that the irreducible representation $\Gamma_{i}$ of degree $n_{i}$ occurs with multiplicity $\mu_{i}^{t}$ in $\left(U^{t}\right)^{-1} R^{t}(\gamma) U^{t}$. The intersections of the $n_{t} \mu_{i}^{t}$ rows and $n_{i} \mu_{i}^{s}$ columns of the block $C^{t s}$ which belong to the representation $\Gamma_{i}$ will be called a representation district $D_{i}^{t s}$. The district itself divides up in to $\mu_{i}^{t} \mu_{i}^{s}$ squares of $n_{i}$ dimensions. With each square is associated a unit matrix $E_{i, k l}^{t s}, k=1, \cdots, \mu_{i}^{t} ; l=1, \cdots, \mu_{i}^{s}$, conveniently denoted by $E_{\eta}^{t s}$. Every matrix permutable with all the reduced matrices $U^{-1} R(\gamma) U$ is a linear combination of these matrices $E_{\eta}^{s}$. In particular we have

$$
\begin{aligned}
U^{-1} V_{\alpha}^{s t} U & =\left(U^{s}\right)^{-1} V_{\alpha}^{s t} U^{t}=\sum_{\eta} \rho_{\alpha \eta}^{s t} E_{\eta}^{s t}, \\
U^{-1} V_{\alpha^{\prime}}^{t s} U & =\sum_{\eta} \bar{\rho}_{\alpha \eta}^{s t} E_{\eta^{\prime}}^{\iota s} .
\end{aligned}
$$

The number of basis matrices $E_{\eta}^{t s}$ for fixed $t, s$, must equal $\mu^{s t}$, the number of $V_{\alpha}^{t s}$, so we have the relation

$$
\mu^{s t}=\sum_{i} \mu_{i}^{s} \mu_{i}^{t} \text {. }
$$

Thus the coefficients $\left(\rho_{\alpha \eta}^{s t}\right)$ defined by (5.1) form a $\mu^{s t}$-dimensional square matrix. Its determinant, denoted by $\mathrm{P}^{s t}$, is not zero, since both the $U^{-1} V^{t_{s}} U$ and the $E^{t_{s}}$ form a basis for the same set of matrices. That $\mathbf{P}^{s t}$ is an algebraic integer (not necessarily rational), divisible by $\left(n^{s} n^{t}\right)^{1 / 2}$, can be shown as follows.

A partial reduction of the linear group to split off the identity representation $\Gamma_{1}$ in each constituent $C^{t}$ can be effected by first replacing $U^{t}$ by a unitary matrix whose $(u, v)$ th element in the block $C^{t t}$ is $\epsilon^{(u-1)(v-1)} /\left(n^{t}\right)^{1 / 2},\left(\epsilon\right.$ an $n^{t}$ th root of unity), and then effecting the reduction in the remaining $n^{t}-1$ dimensions. The coefficients $\rho_{\alpha 1}^{s t}$ are thus readily computed explicitly: 


$$
\begin{aligned}
\rho_{\alpha 1}^{s t} & =\left(g / h_{\alpha}^{s t}\right) /\left(n^{s} n^{t}\right)^{1 / 2}=\left(h^{s} h^{t}\right)^{1 / 2} / h_{\alpha}^{s t} ; \\
\sum_{\alpha} \rho_{\alpha 1}^{s t} & =\sum_{\alpha}\left(n^{s} h^{s} / h_{\alpha}^{s t}\right) /\left(n^{s} n^{t}\right)^{1 / 2}=\left(n^{s} n^{t}\right)^{1 / 2} .
\end{aligned}
$$

Furthermore, since the matrix $W^{t s}$ of (3.8) is of rank 1 and is represented by 0 except in the district $D_{1}^{t s}$, we have

$$
\sum_{\alpha} \rho_{\alpha \eta}^{s t}=0, \quad \eta \neq 1 .
$$

Adding all rows of $\left(\rho_{\alpha \eta}^{s t}\right)$ to the first, we find that

$$
\mathrm{P}^{s t}=\left(n^{s} n^{t}\right)^{1 / 2} \mathrm{P}_{1}^{s t}
$$

where $\mathrm{P}_{1}^{s t}$ is the cofactor of $\rho_{11}^{s t}$ in $\mathrm{P}^{s t}$. Chopping off the first row and column of each, we multiply the conjugate of $\left(\rho_{\alpha \eta}^{s t}\right)$ by the transpose of this same matrix, and obtain a matrix $\left(A_{\alpha \beta}^{s t}\right)$ of $\mu^{s t}-1$ dimensions, with determinant $A^{s t}$ given by the formula

$$
A^{s t}=\overline{\mathrm{P}}_{1}^{s t} \mathrm{P}_{1}^{s t}=\overline{\mathrm{P}}^{s t} \mathrm{P}^{s t} / n^{s} n^{t} \text {. }
$$

The element in row $\alpha-1$ and column $\beta-1$ of $\left(A_{\alpha \beta}^{\text {st }}\right)$ is

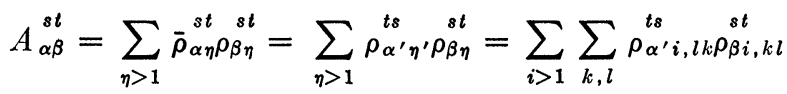

since the subscripts $\eta$ and $\eta^{\prime}$ stand for the three subscripts $(i, k l)$ and $(i, l k)$, respectively. The rule of combination (3.10) which defines the product of $V_{\boldsymbol{\alpha}^{\prime}}^{t s}$ and $V_{\boldsymbol{\beta}}^{s t}$ holds likewise for the representatives of these matrices in the districts corresponding to $\Gamma_{i}$, and we have for fixed $i$

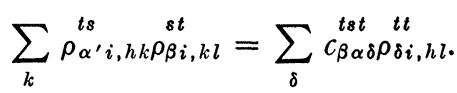

The trace $T_{\delta i}^{t}=\sum_{l} \rho_{\delta i, l l}^{t t}$ of the $\mu_{i}^{t}$-dimensional matrix representing $V_{\delta}^{t t} n_{i}$ times in the district $D_{i}^{t t}$ is an algebraic integer. Hence each element of the matrix $\left(A_{\alpha \beta}^{s t}\right)$,

$$
A_{\alpha \beta}^{s t}=\sum_{\delta, i>1} c_{\beta \alpha \delta}^{t s t} T_{\delta i}^{t}
$$

is an algebraic integer, and the determinant $A^{\text {st }}$ is an integer.

We now use the transformation (5.1) to obtain a new expression for $(4.2)$ :

$$
\begin{aligned}
X_{\alpha \beta}^{s t}(a) & =\chi\left(\sum_{\zeta, \eta} \bar{\rho}_{\alpha \zeta}^{s t} E_{\zeta^{\prime}}^{t s} \rho_{\beta \eta} E_{\eta}^{s t} E_{\eta}^{s t}\left(U^{t}\right)^{-1} R^{t}(a) U^{t}\right) \\
& =\sum_{\eta} \rho_{\beta \eta}^{s t} \bar{\rho}_{\alpha \eta}^{s t} \chi\left(E^{t t}\left(U^{t}\right)^{-1} R^{t}(a) U^{t}\right)=\sum_{\eta} \rho_{\beta \eta}^{s t-s t} \bar{\rho}_{\alpha \eta}^{s t} \chi_{\eta}(a) .
\end{aligned}
$$


Taking determinants of both sides of (5.10) we obtain

$$
\left|X_{\alpha \beta}^{s t}(a)\right|=\mathrm{P}^{s t} \overline{\mathrm{P}}^{s t} \prod_{\eta} \chi_{\eta}(a) \text {. }
$$

The subscript $\eta$ stands for the three subscripts $i, k l$, but since $\chi_{\eta}(a)$ is a character depending on $i$ alone, we group together the $m_{i}=\mu_{i}^{t} \mu_{i}^{s}$ equal factors $\chi_{i}(a)$, use (5.6), and write

$$
\left|X_{\alpha \beta}^{s t}(a)\right|=n^{s} n^{t} A^{s t} \prod_{i}\left[\chi_{i}(a)\right]^{m i} \text {. }
$$

Reference to (4.4) gives us the final formula for the principal theorem of (1.4).

THEOREM.

$$
\begin{aligned}
& \left(n^{t}\right)^{m}\left|K^{s t}\left(\sum a_{\lambda} C_{\lambda}\right)\right|=n^{s} n^{t} A^{s t} \prod_{i}\left(\sum_{\lambda} a_{\lambda} \chi_{i}\left(C_{\lambda}\right)\right)^{\mu_{i}^{s} \mu_{i}^{t}}, \\
& m=\sum \stackrel{\mu_{i} \mu_{i}^{t}=\mu^{s t} .}{m}=
\end{aligned}
$$

6. An illustrative example. To illustrate the application of this theorem to the determination of one of the characters of a fairly complicated "simple" group, we choose the simple group $G_{25920}$, which is an invariant subgroup of index 2 in the group of isomorphisms of the general cubic surface. It can be represented by a group of permutations $R^{1}$ on the 45 tritangent planes (or triangles) of the cubic surface, and also by a group of permutations $R^{2}$ on the 27 lines of the cubic surface. These two transitive groups, when completely reduced, have besides the identity one common irreducible component, whose character we shall compute by our theorem (5.13). Let $H^{1}$ and $H^{2}$ be the subgroups of orders $h^{1}=g / n^{(1)}=576$ and $h^{2}=960$, which leave fixed, respectively, a particular triangle $T$ and one of its lines $L$. The common subgroup of order $h_{1}^{12}=192$ leaves both fixed. There are just two double cosets $H_{\alpha}^{12}$, namely $H_{1}^{12}=H^{1} H^{2} / 192$, consisting of 2880 elements which transform $T$ into one of the five triangles on $L$, and a second double coset $H_{2}^{12}$ consisting of 23040 elements which transform $T$ into one of the other 40 triangles. Hence there are just two irreducible components common to $R^{1}$ and $R^{2}$, the identity and one other. There are three double cosets $H_{\alpha}^{11}$, namely $H_{1}^{11}=H^{1}$ containing 1 coset leaving $T$ fixed, $H_{2}^{11}$ containing 12 cosets which take $T$ into one of the 12 "adjacent" triangles having a line in common with it, and $H_{\mathbf{3}}^{11}$ containing 32 cosets taking $T$ into a non-adjacent triangle.

To determine the constants $c_{\alpha \beta \eta}^{t s t}$, we first note that

$$
H_{1}^{12} H_{1}^{21} / 960=3 H_{1}^{11}+H_{2}^{11},
$$


since just $1 / 5$ of the operations of $H^{1} H^{2}$ belong to $H^{1}$ and the other $4 / 5$ transform $T$ into an adjacent triangle, and since $H_{2}^{11}$ contains 12 times as many elements as $H_{1}^{11}$. Then we use the formulas (3.12), which become

$$
\begin{aligned}
& \sum_{\alpha} c_{\alpha 1 \eta}^{121}=\sum_{\alpha} c_{1 \alpha \eta}^{121}=h^{1} / h_{1}^{12}=3 \\
& \sum_{\alpha} c_{\alpha 2 \eta}^{121}=\sum_{\alpha} c_{2 \alpha \eta}^{121}=h^{1} / h_{2}^{12}=24 .
\end{aligned}
$$

The matrices $M^{s}\left(H_{\eta}^{11}\right)$ may now be written

$$
\begin{gathered}
M^{2}\left(H_{1}^{11}\right)=\left(\begin{array}{ll}
3 & 0 \\
0 & 24
\end{array}\right), \quad M^{2}\left(H_{2}^{11}\right)=\left(\begin{array}{lr}
1 & 2 \\
2 & 22
\end{array}\right), \\
M^{2}\left(H_{3}^{11}\right)=\left(\begin{array}{rr}
0 & 3 \\
3 & 21
\end{array}\right) .
\end{gathered}
$$

In factoring the determinant $\left|K^{21}\right|$ of (1.3) it will be convenient to omit the superscripts from $b_{\eta}^{1}, k_{\eta \lambda}^{1}, g_{\eta \lambda}^{1}, A^{21}$. Then

$$
\begin{aligned}
\left|K^{21}\right| & =\left|\begin{array}{cc}
3 b_{1}+b_{2} & 2 b_{2}+3 b_{3} \\
2 b_{2}+3 b_{3} & 24 b_{1}+22 b_{2}+21 b_{3}
\end{array}\right| \\
& =\left(b_{1}+b_{2}+b_{3}\right)\left(72 b_{1}+18 b_{2}-9 b_{3}\right) .
\end{aligned}
$$

The first of these factors corresponds to the identity representation $\Gamma_{i}$. The other factor remains to be investigated. Since $n^{1}=45$, equation (5.13) gives

$$
(45)^{2}\left(72 b_{1}+18 b_{2}-9 b_{3}\right)=27 \cdot 45 A \sum_{\lambda} a_{\lambda} \chi\left(C_{\lambda}\right)
$$

where

$$
b_{\eta}=\sum_{\lambda} a_{\eta} k_{\eta \lambda}, \quad A=\mathrm{P}_{1}^{21} \overrightarrow{\mathrm{P}}_{1}^{21}
$$

Equating coefficients of $a_{\lambda}$ in (6.5) after using (6.6) we have

$$
A \chi\left(C_{\lambda}\right)=(5 / 3)\left(72 k_{1 \lambda}+18 k_{2 \lambda}-9 k_{3 \lambda}\right) \text {. }
$$

The degree $d$ of this representation is $d=120 / A$, which must be an integer less than 27. Noting that $k_{1 \lambda}=g_{1 \lambda}, k_{2 \lambda}=12 g_{2 \lambda}$, and $k_{3 \lambda}=32 g_{3 \lambda}$, we may rewrite (6.7) in the form

$$
\begin{aligned}
\chi\left(C_{\lambda}\right) & =d\left(g_{1 \lambda}+3 g_{2 \lambda}-4 g_{3 \lambda}\right) \\
& =g_{\lambda} \chi_{\lambda}
\end{aligned}
$$


where $\chi_{\lambda}$ is the trace of a single element of $C_{\lambda}$ and $g_{\lambda}$ is the number of elements in the class $C_{\lambda}$. To complete the computation it is necessary to find the constants $g_{\eta \lambda}$. Taking the 20 classes of conjugates of the simple group of order 25920 in the order listed in an earlier paper, ${ }^{4}$ we calculate $\chi\left(C_{\lambda}\right)$ and $\chi_{\lambda}$ as follows.

\begin{tabular}{|c|c|c|c|c|c|c|c|}
\hline$g_{\lambda}$ & $g_{1 \lambda}$ & $g_{2 \lambda}$ & $g_{3 \lambda}$ & $\chi\left(C_{\lambda}\right)$ & \multicolumn{3}{|l|}{$\chi_{\lambda}$} \\
\hline 1 & 1 & 0 & 0 & $d$ & $d$ & $=$ & 20 \\
\hline 45 & 13 & 0 & 1 & $9 d$ & $d / 5$ & $=$ & 4 \\
\hline 270 & 30 & 12 & 3 & $54 d$ & $d / 5$ & $=$ & 4 \\
\hline 540 & 12 & 12 & 12 & 0 & 0 & $=$ & 0 \\
\hline 40 & 8 & 0 & 1 & $4 d$ & $d / 1$ & $0=$ & 2 \\
\hline 40 & 8 & 0 & 1 & $4 d$ & $d / 1$ & $0=$ & 2 \\
\hline 360 & 8 & 0 & 11 & $-36 d$ & $-d / 1$ & $0=-$ & 2 \\
\hline 360 & 8 & 0 & 11 & $-36 d$ & $-d / 1$ & $0=-$ & -2 \\
\hline 2160 & 48 & 48 & 48 & 0 & 0 & $=$ & 0 \\
\hline 2160 & 48 & 48 & 48 & 0 & 0 & $=$ & 0 \\
\hline 480 & 32 & 0 & 14 & $-24 d$ & $-d / 2$ & $0=-$ & -1 \\
\hline 1440 & 32 & 48 & 26 & $72 d$ & & $0=$ & 1 \\
\hline 240 & 32 & 12 & 2 & $60 d$ & $d /$ & $4=$ & 5 \\
\hline 2160 & 96 & 60 & 42 & $108 d$ & & $0=$ & 1 \\
\hline 720 & 64 & 12 & 16 & $36 d$ & $d / 2$ & $0=$ & 1 \\
\hline 720 & 64 & 12 & 16 & $36 d$ & $d / 2$ & $0=$ & 1 \\
\hline 3240 & 72 & 72 & 72 & 0 & 0 & $=$ & 0 \\
\hline 5184 & 0 & 144 & 108 & 0 & 0 & $=$ & 0 \\
\hline 2880 & 0 & 48 & 72 & $-144 d$ & $-d / 2$ & $0=-$ & -1 \\
\hline 2880 & 0 & 48 & 72 & $-144 d$ & $-d / 2$ & $0=-$ & -1 \\
\hline
\end{tabular}

The scalar product of the columns $\chi\left(C_{\lambda}\right)$ and $\chi_{\lambda}$ is equal to the order 25920 of the group, so we have $1296 d^{2} / 20=25920 ; d=20 ; A=6$. Hence the degree $d$ is 20 , and the last column gives the required character of the irreducible representation of this degree. Eliminating the common components of degree 1 and 20 from the permutations on 45 and 27 symbols, we immediately obtain two other irreducible components of degree 24 and 6 , respectively. The components of degree 1 and 20 occur in the Kronecker square of the one of degree 6 , and an irreducible component of degree 15 is left. Thus the laborious work of obtaining the character of degree 20 bears fruit in obtaining three more characters with almost no work at all. The theorem of this paper gives a method of digging out some of the characters of a group if simpler methods are not available.

BROWN UNIVERSITY

${ }^{4}$ J. S. Frame, The simple group of order 25920, Duke Math. J. vol. 2 (1936) p. 483. 